\title{
PLEIADES-HR INNOVATIVE TECHNIQUES FOR GEOMETRIC IMAGE QUALITY COMMISSIONING
}

\author{
D. Greslou, F. de Lussy, J.M. Delvit, \\ C. Dechoz, V. Amberg
}

CNES 18, avenue Edouard Belin 31401 TOULOUSE CEDEX 4 France

Phone: 33.(0)5.61.27.39.97 Fax: 33.(0)5.61.27.31.67 E-mail: daniel.greslou@cnes.fr

\section{ISPRS and IAA : Pléiades Inflight Calibration and Performance Assessment}

KEY WORDS: Image Quality, Geometry, Calibration

\begin{abstract}
:
Since the beginning of 2012, the first Pleiades-HR satellite of the program conducted by the French National Space Agency, CNES, delivers $20 \mathrm{~km}$ wide color scenes with a $70 \mathrm{~cm}$ ground sampling distance. A second satellite should be launched in 2013 which will achieve an almost world-wide coverage with a revisit interval of $24 \mathrm{~h}$.

The assessment of the image quality and the calibration operation have been performed by CNES Image Quality team during the 6 month commissioning phase that followed the satellite launch. The geometric commissioning activities consist in improve the geometric quality of the images in order to meet very demanding specifications as localization accuracy, local coherence, dynamic stability, length alteration ... This goal has been achieved through the implementation of new methods of calibration and performance assessment. Some of these methods are based on the exploitation of very specific satellite acquisitions that have been achieved thanks to the amazing agility of the Pleiades satellite. Thus, many stars acquisitions and very slow earth pictures have been processed to characterize dynamic phenomena. Similarly, "along-cross track" pairs have been exploited to improve the accuracy of the focal plane description. This paper deals with these new methods. It describes their accuracy and their operational interests.
\end{abstract}

\section{INTRODUCTION}

The in-orbit commissioning phase aims to calibrate some parameters to achieve the best quality on images. In geometry, these activities are mainly conducted in order to finely characterize the acquisition model, needed to ensure the location, multispectral registration and internal consistency performances of images.

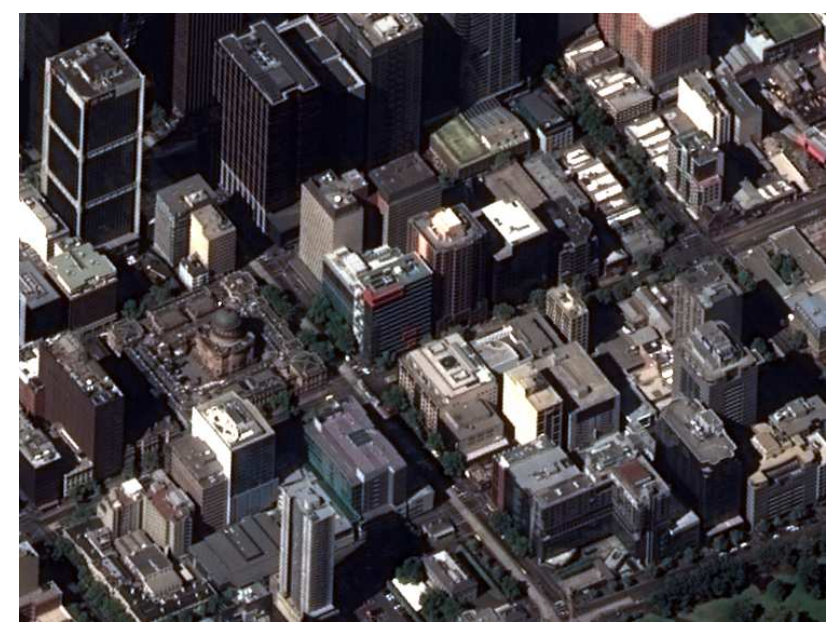

Figure 1 : Pleiades-HR image of Melbourne downtown, (C) CNES Copyright 2012

This paper describes the main geometric calibrations implemented on the satellite PHR-1A during the first phase of in-orbit commissioning led by the CNES [13]. They consist principally in asses the main biases of the location model to improve its accuracy and achieve an accurate cartography of the focal plane in order to improve registration of the different bands and correct local distortions.

We have deliberately focused on the description of new and innovative methods, that were made possible thanks to the great agility of the satellite. We have also pointed out the accuracy of these methods and given an estimation of the main geometric performance reached by the satellite after three months of inorbit settings. All these results must be consolidated, but they prefigure the final geometric quality image performances of the system Pleiades.

\section{GEOMETRIC CALIBRATION ACTIVITES}

\subsection{Viewing reference frame biases calibration}

These main biases are the sum of all instruments and platform biases that impact the location model. They are related to the instrument (viewing) reference frame. These biases are classically evaluated during in-orbit commissioning with numerous measurements made on a worldwide GCP basis. This analysis is conducted on a time horizon of several months, allowing a characterization of the behavior of these biases over time and a modelling of orbital and seasonal variations that would impact localization performance [10]. This method is very accurate and reliable but needs a broad base of GCP which availability and access are not always easy. Moreover, accuracy of each GCP has to be consistent with the requirements of the system location performance (typically on PHR, accuracy of the GCP is better than $1 \mathrm{~m})$.

An innovative method, called "auto-reverse" was tested on PHR during the commissioning phase [11]: this was made possible thanks to the extreme agility of the satellite. Indeed, from a couple of images acquired from the same orbit with 
inverse directions as shown on figure 2 , we can asses the roll and pitch biases without the use of exogenous data.

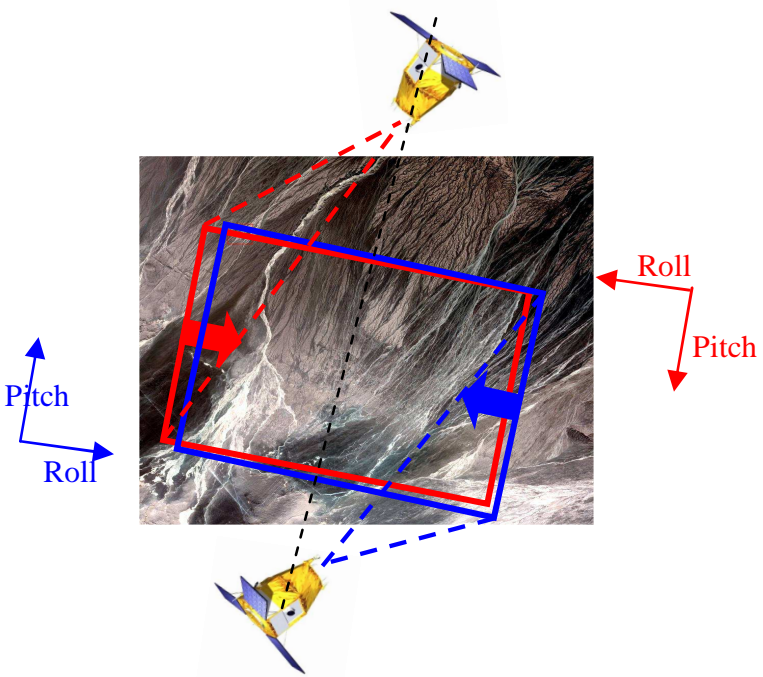

Figure 2: "Auto-Reverse" acquisition principle

The U-turn made by the satellite between the two acquisitions causes an inversion of instrument axes: the relative location offset between the two images can be calculated: it is representative of twice the value of the bias referred on each axis.

This method has the advantage that it can be implemented anywhere on the world, and in particular on places where there are no baseline data, but also, from few solicited portions of orbit.

The results of this method are consistent with those assessed by exploiting conventional GCP. Its accuracy depends mainly on the quality of the alignment of two images which allows considering that roll and pitch axes are inversed. Moreover, it is better to make these acquisitions on relatively flat areas to minimize the impact of DEM error. This method is particularly suitable for roughly estimating the location bias and for monitoring their evolution.

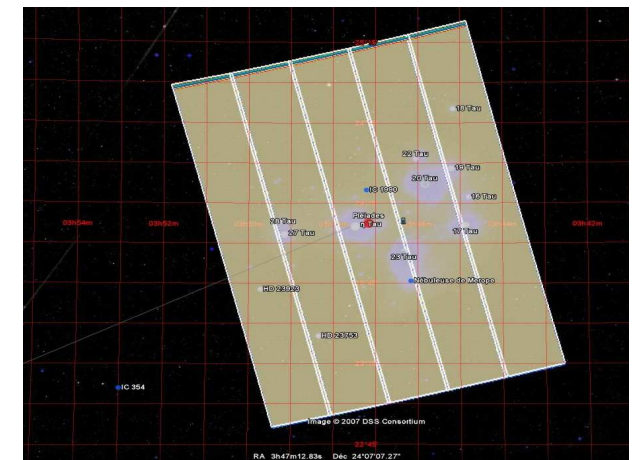

Figure 3 : Example of zone of interest: the Pleiades constellation

A third method began also to be tested. It consists in exploit the stars of the sky as measurements very accurate. The $2^{\circ}$ angular PHR field of view allows imaging several stars from various constellations [14]. Similarly to Earth acquisitions, each star can be considered as a GCP to estimate biases. The huge advantage of this method is that these acquisitions are scheduled during night orbit without interfering with the commercial programming and with the absolute certainty of avoiding the clouds!

A peculiarity of this method lies in the consideration of the effect-called "relativistic aberration". [4] describes this phenomenon and analyzes its impact on the location model. This effect is similar to a bias whose magnitude and direction depend on the relative velocity between the observer and the object observed. For "Earth" acquisitions, for instance, only the orbital motion of the satellite must be taken into account in the calculation, while for stellar observations, we must also take into account the Earth's orbital motion.

By exploiting stellar acquisitions, estimated biases can differ than those assessed by conventional methods which uses GCP. We must therefore take into account properly this effect to avoid distorting the calculation of the earth location model bias. This method has not yet been implemented operationally. The tests were mainly done to validate the principles and validate the operational feasibility for the future systems.

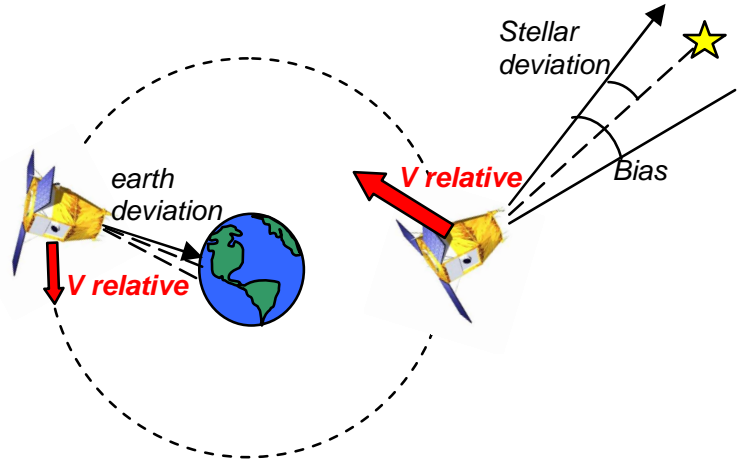

Figure 4: Light-aberration principle

The calibration of the yaw bias is a little more difficult because of a lever arm 70 times lower than the previous biases. For a field of $20 \mathrm{~km}$, the impact of a yaw error of $10 \mu \mathrm{rad}$ corresponds to an overall rotation of the field of about $10 \mathrm{~cm}$ at the ends which is very difficult to observe from a very few GCP pointed in the image. To improve the observability of the yaw bias, we increased the lever arm by operating a multi-pass coverage of $200 \mathrm{~km}$ wide, consisting of approximately 10 PHR segments.

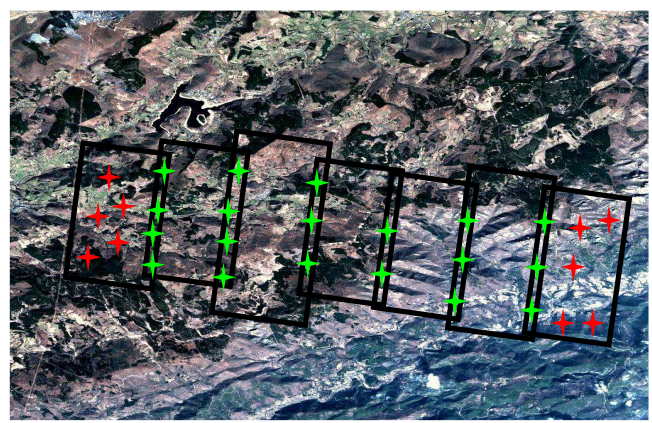

Figure 5 : Multi-pass coverage

These segments are then used in a block of space-triangulation previously equipped with ground control points (at the ends) and tie points in the overlap areas as shown in the figure 5. This 
process allows estimating a yaw bias but also a focal length correction with enough accuracy.

These biases are analyzed closely during all commissioning phase [10] in order to highlight temporal tendencies that could be incorporated into the location model.

\subsection{Focal Plane calibration}

The focal plane cartography or mapping, which means the assessment of the accurate viewing directions of every pixel in the instrumental frame, is required to obtain a good registration of all spectral bands but also to improve the internal geometric consistency of the images by correcting local distortions.

The PHR focal plane is composed of 5 detector arrays for PA and MS bands placed end to end with an overlapping InterArray-Zone (IAZ) to ensure line continuity. The processing of orthoimage and perfect sensor products ([1]) requires a fairly accurate calibration of the focal plane, in particular to ensure a perfect "mosaicking" of the 5 elementary images and a perfect registration needed for the fusion of spectral bands.

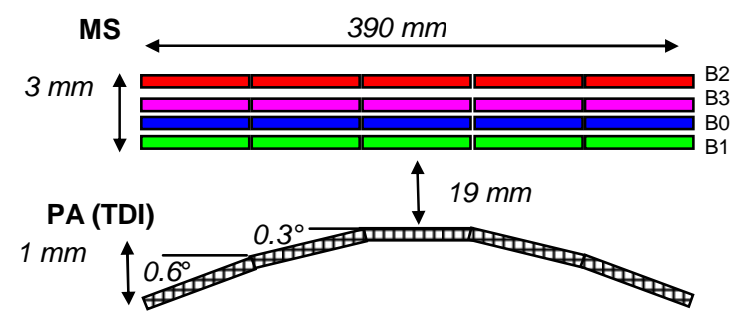

Figure 6: PHR Focal plane layout

The classical calibration method is based on exploitation of a reference image called "Super-Site" of high geometric quality. The imagery used for the PHR calibration is the result of an airborne campaign covering a $20 \times 20 \mathrm{~km}^{2}$ wide area. The image was acquired in summer 2009 and is centered on the city of Toulouse. The super-site product is composed of a $12 \mathrm{~cm}$ orthoimage and a fairly accurate $1 \mathrm{~m}$ DEM generated by photogrammetric calculation. These data are used in order to simulate a reference image on the PHR focal plane which is compared to the PHR acquisition by dense matching process from which we can deduce PA viewing directions model corrections. The graphs of the figure 7 below, represent the model corrections obtained by averaging the correlation grids.

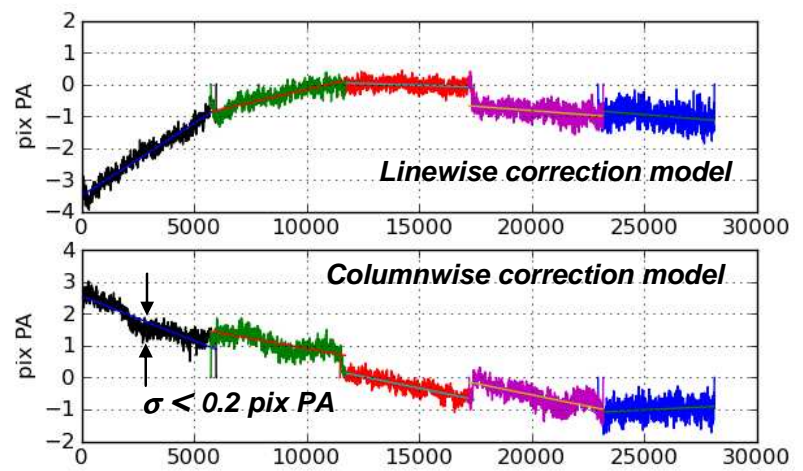

Figure 7 : PA focal plane cartography in-orbit corrections
The accuracy is quite good but a little noisy (rms $<0.2$ PA pixel) due to important radiometric differences between the 2 years old summer reference image and the recent winter PHR acquisition (cf. figure 8) which are illustrated on pictures below.

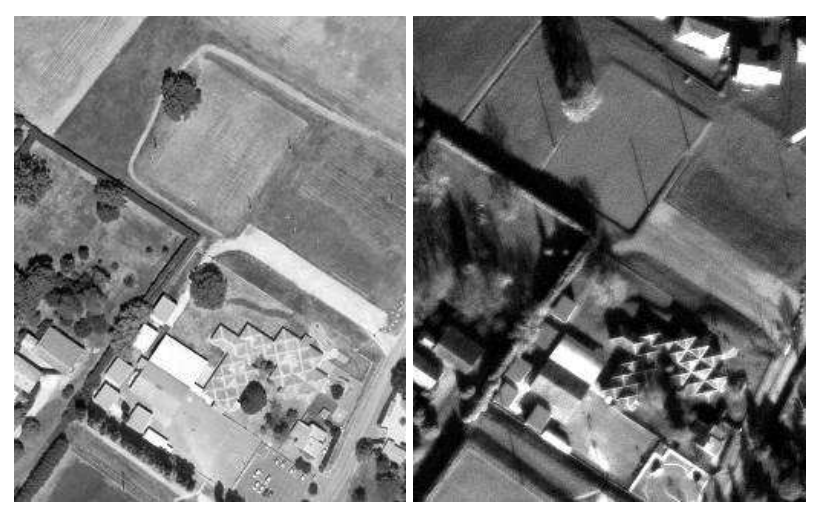

Figure 8 : Radiometric differences between image reference and PHR acquisition

However, accuracy is enough to fulfill PHR specifications.

A special care had also be taken to the calibration of the IAZ mapping in order to make transitions between array undetectable in orthoimage and perfect sensors products [1]. Indeed, a radiometric reconstruction process is applied in these areas, requiring a very precise relative mapping. This is done by exploiting a particular stereo pair acquisition as shown on figure 9. The longitudinal shift between the two acquisitions allows an accurate characterization of the IAZ of one image by exploiting the other image and vice versa.

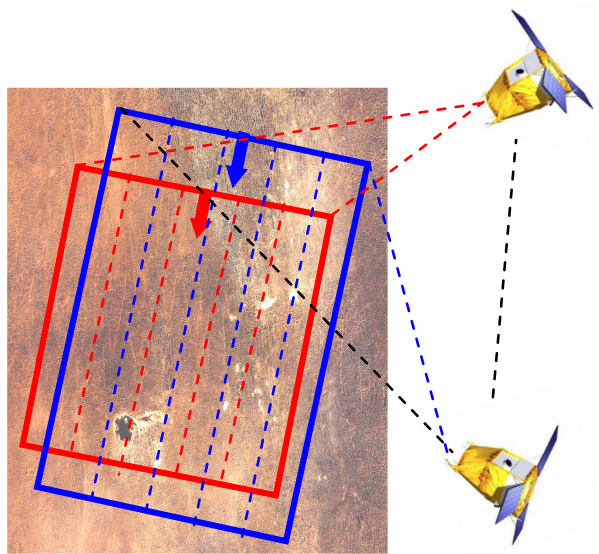

Figure 9 : IAZ calibration stereo-pair acquisition principle

A rather innovative method to calibrate the focal plane has also been tested and compared to the conventional method. This is the called "cross-mode" method. It consists in exploit a couple of images acquired with orthogonal directions.

The properties of the push-broom acquisition allow separating static and temporal aspects along the rows and columns of the image. The two images are correlated and statistical computation is applied on the lines and columns of the disparity map. Thus, one can succeed in finely characterize the absolute focal plane of an image by averaging the temporal aspects of the other and vice versa. 
The major advantage of this method is that it allows performing various absolute focal plane mapping anywhere in the world which would be very costly by exploiting classic methods based on reference sites. Moreover, the quality of the correlation process of such a couple is much better than that obtained by exploiting a reference picture because it doesn't present significant radiometric differences due to changes in the landscape, which strongly degrade the performance of correlation and thus the accuracy of the calibration. With this method, the calibration noise is reduced by a factor of 2 with respect to the conventional method [11].

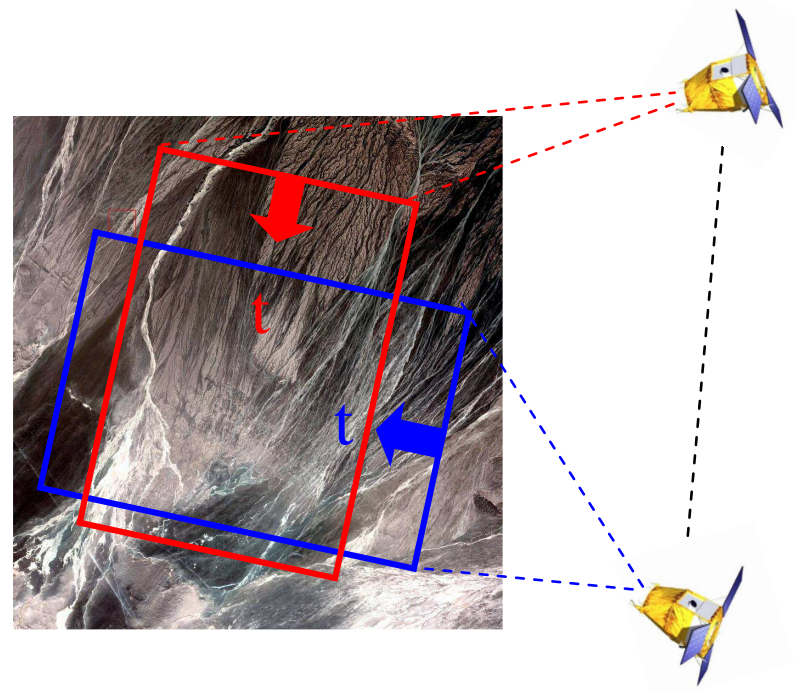

Figure 10 : "Cross-mode” acquisition principle

Finally, the mapping of the MS viewing directions is then performed using the panchromatic image as a reference (once it is calibrated!). It is this last step that guaranties correct registration between all spectral bands. The process consists again in a very dense matching process of every spectral band in order to identify corrections. This method has the advantage of being implemented on a multitude of images, different types of landscapes and in different conditions of acquisition. We obtain a very accurate characterization of MS viewing directions with respect to $\mathrm{PA}$.

After first calibration performed during commissioning, the registration residuals are currently less than 0.25 pixel MS which is much better than the specifications. Efforts continue to improve this performance.

\subsection{Stability calibration}

The PHR satellite has great agility thanks to its gyroscopic actuators (CMG) that provide a very high torque enabling fast rotations [12]. The torque is obtained by varying the rotational axis of a wheel spinning at constant rate. This rate must be chosen carefully to ensure maximum agility of the satellite while minimizing impacts in terms of micro-vibration of the line of sight. Indeed, a very specific rate can cause harmonic disturbances that can be transmitted through the structure until the instrument and degrade image quality.

Several methods were used to conduct the dynamic analyses and choose the spin rate best suited to the actuators.

The first method is the most representative of actual conditions of acquisition. It consists in matching the different bands MS to each other. It is similar to the focal plane calibration but instead doing a static analysis by calculation of a mean row, the disparity maps are averaged along the other direction providing temporal information. The MS detectors are placed parallel to each other (fig 5). The acquisition of the landscape is done with a temporal delay of about $7 \mathrm{~ms}$ between two consecutive band. By correlating the images, we can highlight distortions caused by disturbances during this time interval. This principle is behind the processing described in [14] which estimates vibrations on images.

The advantage of this method is that it allows detailed characterization of the disturbances in both directions, line and column (i.e. pitch and roll). Furthermore, this analysis can be conducted when the acquired image has good ability to correlate.

Another method consists in make an acquisition on Earth's surface by steering the satellite so that the projection of the scan-line on the ground remains constant along the image as shown on figure 11. It is the called steady-mode".

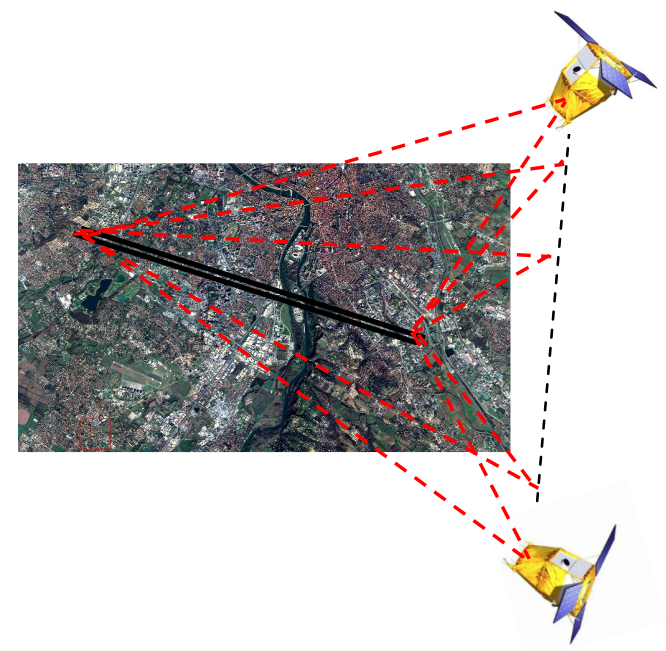

Figure 11: «Steady-mode » acquisition principle

The offset in column assessed by matching techniques between two consecutives lines (cf. figure 12) is representative of a roll disturbance. It allows determining a vibration profile characteristic of the CMG spin rate.

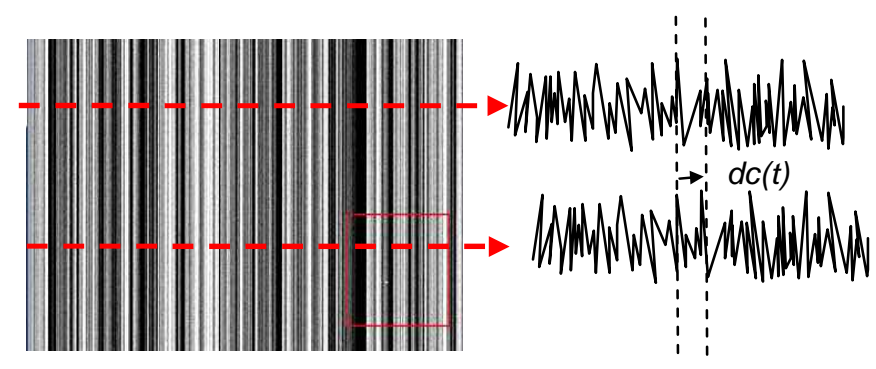

Figure 12 : "Steady-mode" image extract

A third method was preferred to the latter and has been widely used in several campaigns acquisitions: it is the method called "StarAcq" described in [6], [8], [13], [14]. It is similar to the "steady-mode", except that in this case, the satellite operates a slow-motion, not on the Earth's surface but on a star.

The main advantage of the method is that it uses images that may be acquired on the orbits of night avoiding overloading operational/commercial programming made during days orbit. 
The main difficulty with this method is to image the star with the "narrow" aperture of the TDI matrix (only maximum 20 stages!). Indeed, the star being a point source, it is very difficult to match acquisition of the very thin detector with the star. To be sure to acquire the star, a specific guidance, called "StarAcq" allows covering part of the sky and absorbing the pointing accuracy. The drift of the guidance applied during the acquisition limits the duration of such images on which the star is visible only on a few thousand rows but rather enough to carry out dynamic analysis.
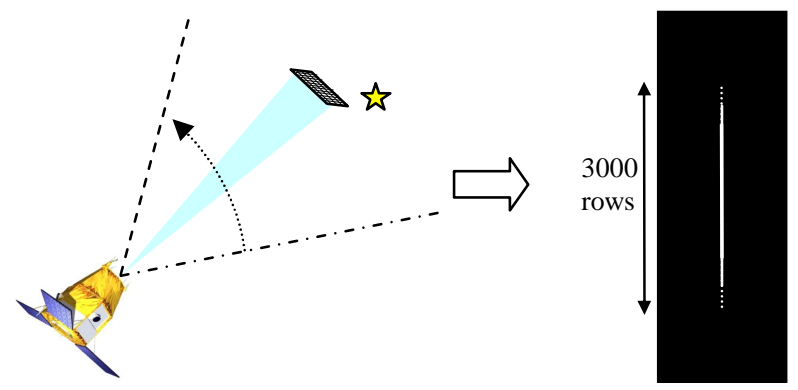

Figure 13 : Staracq acquisition principle and image extract

The exploitation of such acquisitions is similar to that carried out on "steady images". A line by line matching process can reveal disturbances along the lines due to a roll vibration. This method is very efficient and accurate. It has allowed the identification of disturbances of very low magnitude (until $10^{-9}$ rad!) and to choose the most appropriate rate spin for the actuators wheels very rapidly.

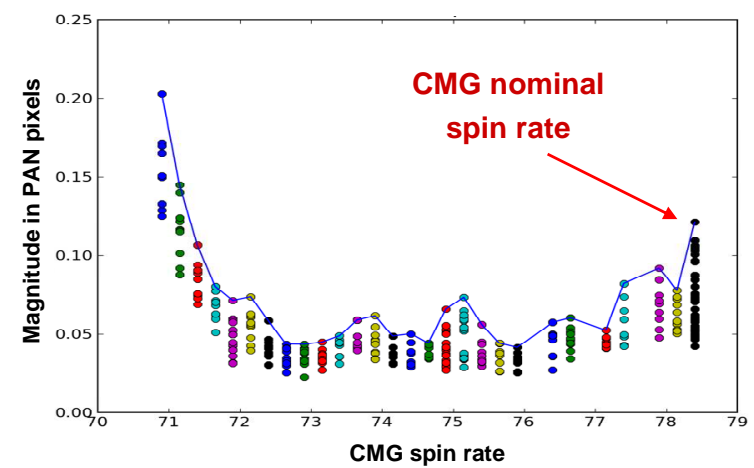

Figure 14 : maximum micro-vibrations magnitude vs CMG spin rate

All dynamic analyzes did not reveal the presence of vibratory phenomenon impacting the quality of images. Dynamic disturbances remains at levels much lower than the system specification (0.2 PA pix) and CMG spin rate has been chosen to preserve best agility.

\section{MAIN GEOMETRIC IMAGE QUALITY PERFORMANCES}

After three months of calibration activities, PHR geometric performances meets its planned specifications. The agility of the satellite allows testing new innovative techniques very successfully. These will be implemented without reserve on the next satellites and in particular on PHR-1B.
The table below gives the main image quality performances assessed between launch and 2012 April, $1^{\text {st }}$. Some of them should be enhanced by the end of the commissioning phase scheduled 2012, June $30^{\text {th }}$.

\begin{tabular}{|l|c|}
\hline Image Quality criteria & Performance \\
\hline Localization CE90 & $16 \mathrm{~m}$ \\
\hline PA/MS registration in MS pixels & 0.25 \\
\hline MS/MS registration in MS pixels & 0.15 \\
\hline Planimetry in PA pixels & 0.20 \\
\hline
\end{tabular}

Table 1. Main Geometric Image Quality performances assessed 2012, April, $5^{\text {th }}$.

These results are still consolidated, but they prefigure the final geometric quality image performances of the system Pleiades.

\section{REFERENCES}

[1] F. de Lussy \& al, "Pleiades-HR Images System products and quality Pleaides-HR Image syqtem products and geometric accuracy", ISPRS Hannover (2005)

[2] J-M. Delvit \& al, "The geometric supersite of Salon de Provence", ISPRS Congress Paris (2006).

[3] L. Lebègue \& al, "Using Exotic Guidance for PLEIADESHR Image Quality Calibration,” ISPRS Beijing (2008).

[4] D. Greslou \& al, "Light aberration effect in HR geometric model", ISPRS Beijing (2008).

[5] F. de Lussy \& al, "Process line for geometrical image correction of disruptive microvibrations", ISPRS Beijing 2008

[6] S. Fourest \& al, "Star-Based Calibration Techniques for PLEIADES-HR Satellites", CALCON Logan (2009).

[7] JM. Delvit \& al, "Geometric improvement for Earth observation applications", IGARSS Honolulu (2010).

[8] L. Lebègue \& al, "Pleiades-HR Image Quality commissioning foreseen methods", IGARSS Honolulu (2010).

[9] P. Kubik \& al, "First in-flight results of Pleiades 1A innovative methods for optical calibration", ICSO Ajaccio (2012).

[10] F. de Lussy \& al, "Pleiades HR in flight geometrical calibration : Localisation and mapping of the focal plane", ISPRS Melbourne (2012).

[11] JM. Delvit \& al, "Attitude assessment using Pléiades-HR capabilities", ISPRS Melbourne (2012)

[12] A. Gleyzes \& al, "Pleiades system architecture and main performances", ISPRS Melbourne (2012)

[13] L. Lebegue \& al, "Pleiades-HR image quality commissioning", ISPRS Melbourne (2012).

[14] S. Fourest \& al, "Star-based methods for Pleiades HR commissioning”, ISPRS Melbourne (2012). 